\title{
La limitación de la producción de los aceites de oliva por parte del estado español
}

\section{Introducción}

El sector agroalimentario, esto es, el conjunto de los sectores productivos (agrícola, ganadero, forestal y pesquero) que se dedican a producir, transformar y distribuir sus productos en el mercado ${ }^{1}$, presenta una situación bastante complicada en lo que se refiere a la inestabilidad de los precios en origen. Con cierta frecuencia observamos cómo los precios que recibe el productor por la materia prima están por debajo del coste real de producción. No sucede lo mismo con el último eslabón de la cadena, el de la comercialización, que normalmente obtiene beneficios por la venta de dichos productos salvo que se lleve a cabo, la venta a pérdidas ${ }^{2}$, lo que conlleva la realización en el mercado de prácticas lesivas del derecho de la competencia.

Por su parte, el sector del aceite de oliva es uno de los principales en cuánto a producción agroalimentaria que sufre este tipo de desequilibrios

* Universidad de Jaén, España.

${ }^{1}$ Art. 5 b) de la Ley 12/2013, de 2 de agosto, de medidas para mejorar el funcionamiento de la cadena alimentaria. Boletín Oficial del Estado (BOE) núm. 185, de 3 de agosto de 2013.

${ }^{2}$ El Art. 17 de la Ley 3/1991 de Competencia Desleal, dispone con carácter general, la libre fijación de precios en el mercado pero determina una serie de circunstancias que determinan si la fijación de un precio bajo costo de adquisición es en sí un acto de competencia desleal. Los requisitos al respecto hacen referencia al que precio induzca error sobre otros productos y servicios en el mismo establecimiento, que desacredite la imagen del producto o de un establecimiento ajeno o que tenga como objetivo la eliminación de un competidor en el mercado. 
de forma recurrente. Conviene precisar que el sector del olivar, además de estar presente en casi todas las regiones de España, es el sustento principal de la economía de la provincia de Jaén como líder de la producción mundial y tiene igualmente una función social importante como es la de impedir el abandono de las zonas rurales y fijar la población al territorio.

Además, la producción de aceite de oliva, al igual que el resto del sector primario, está principalmente condicionada por el clima, lo que supone una clara diferencia entre las distintas campañas oscilando entre los 1,4 y 1,8 millones de toneladas a nivel mundial. Por otra parte esto supone que los precios sufran una gran volatilidad con una fuerte repercusión no sólo en la perduración de los territorios productores, sino también en la viabilidad económica de las empresas productoras.

A los problemas que presenta el cambio climático para la producción, habría que añadir que la mayor parte de la superficie dedicada a este cultivo es tradicional y en secano, es decir, árboles de dos y tres pies centenarios difícilmente mecanizables y sin posibilidad en su mayoría de acceder al agua. A ello se suma que a lo largo de los últimos años se está incrementando la superficie de olivar con plantaciones intensivas o superintensivas cuyo coste de producción es inferior al del olivar tradicional no así la productividad que es sensiblemente superior, lo que hará que aumente la obtención de aceite de oliva mientras la demanda se mantiene estable a nivel mundial.

Por último, el mercado oleícola de nuestro país, está claramente desequilibrado en cuanto al número de compañías que ofrecen y demandan este tipo de productos. Por una parte, existen numerosas empresas productoras como cooperativas o almazaras que ofrecen aceites de oliva al mercado, sin embargo, muy pocas que lo envasen y lo pongan en comercialización, lo que supone una clara desventaja para las primeras en lo que se refiere al poder de negociación. Esto no afecta por igual a todos los niveles de la cadena agroalimentaria ya que normalmente se producen desequilibrios entre las transacciones que se producen entre operadores que tienen escasa cuota de mercado pero no así con compañías que aglutinan un mayor número de producción. Resulta evidente que en este mercado desequilibrado entre los que ofrecen y demandan, se muestra perjudicado aquél que suma mayor número ya que los demandantes se benefician de un nivel muy alto de competencia, algo que podría ser beneficioso para este eslabón de la cadena y para el consumidor pero no así para el sector productor.

Por todo ello, hemos puesto de manifiesto que se trata de un sector que por las distintas circunstancias que le rodean, va encaminado de forma constante al desequilibrio en cuanto a los precios en el mercado nacional. Ante estas circunstancias cabría plantearse si puede el legislador aprobar alguna norma que permita estabilizar los precios en origen mediante el control o la 
limitación de la producción y si la adopción de dicha norma va a provocar la falta de competencia en el mercado. Pues bien, el legislador ha optado por aprobar mediante Real Decreto, el establecimiento de las normas básicas que permitan de forma ordenada retirar la producción del mercado al objeto de paliar la situación precaria sobre todo en los precios y lo ha hecho basándose en una aplicación de un reglamento europeo. A continuación analizaremos, desde la perspectiva del Derecho de la competencia, si la norma se asimila o no, a la práctica de los acuerdos colusorios o si, por el contrario, permitirá que siga existiendo competencia en el mercado del sector oleícola español.

\section{El análisis de las excepciones previstas en las normas de competencia españolas sobre acuerdos colusorios}

El ordenamiento jurídico español cuenta con una norma, la Ley 15/2007 de Defensa de la Competencia ${ }^{3}$, que regula el nivel de competencia entre las empresas en nuestra economía y controla el buen funcionamiento del propio mercado en lo que se refiere al comercio de bienes y servicios. Por su parte, a nivel comunitario, el Tratado de Funcionamiento de la Unión Europea (TFUE) establece que "la Unión definirá y aplicará una política común de agricultura y pesca"4 con el objetivo, entre otros, de estabilizar lo mercados y garantizar un nivel de vida a la población agrícola ${ }^{5}$. Estos objetivos están definidos en el Reglamento Europeo por el que se crea la organización común de mercados de los productos agrícolas ${ }^{6}$ (en adelante OCM) que establece una serie de normas comunes sobre la competencia. Dicha norma fomenta además, la constitución de organizaciones de productores con el fin de estimular el trabajo conjunto y mejorar la posición en la cadena de los agricultores ${ }^{7}$.

Ahora bien, en ningún caso el objeto de esta legislación, es implantar un mercado en competencia perfecta, aún siendo éste un modelo ideal, ya que esto sería completamente irrealizable por multitud de factores. Desde nuestro punto de vista, la práctica de un mercado ideal supondría para el caso que nos ocupa una opción perjudicial al afectar al eslabón de la cadena más débil, de ahí que se tengan en cuenta otras razones de carácter socioeconómico para

${ }^{3}$ BOE núm. 159, de 4 de julio de 2007.

${ }^{4}$ Art. 38 del TFUE.

${ }^{5}$ Art. 39 del TFUE.

${ }^{6}$ Reglamento (UE) No 1308/2013 del Parlamento Europeo y del Consejo de 17 de diciembre de 2013 por el que se crea la organización común de mercados de los productos agrarios y por el que se derogan los Reglamentos (CEE) no 922/72, (CEE) no 234/79, (CE) no 1037/2001 y (CE) no $1234 / 2007$.

${ }^{7}$ Art. 152 del Reglamento (UE) no 1308/2013, del Parlamento Europeo. 
que el consumidor pueda seguir disfrutando de ciertos bienes y servicios especialmente en el sector agroalimentario.

El Reglamento OCM reconoce la posibilidad a una organización de productores de aceite de oliva reconocida conforme a la norma, la facultad de negociar los contratos de suministros con el objetivo de concentrar la oferta u optimizar $\operatorname{costes}^{8}$. Ahora bien, siempre que el volumen de la producción de aceite de oliva no supere el 20 por ciento del mercado relevante 9 . Esta opción que resulta claramente ventajosa para el sector no produce el efecto deseado en ocasiones en el ámbito de la producción del aceite de oliva al ser insuficiente para estabilizar el mercado.

De otro modo, la norma sobre defensa de la competencia nacional, establece además tres conductas prohibidas. En primer lugar, el veto a las prácticas colusorias ${ }^{10}$; en segundo lugar, la práctica de abuso de la posición dominante ${ }^{11} ; \mathrm{y}$, por último, prohíbe el falseamiento de la competencia mediante actos desleales ${ }^{12}$. La primera de ellas, esto es, las prácticas colusorias, se refieren a la prohibición de "todo acuerdo, decisión o recomendación colectiva, o práctica concertada o conscientemente paralela, que tenga por objeto, produzca o pueda producir el efecto de impedir, restringir o falsear la competencia en todo o parte del mercado nacional". A priori, analizando el contenido de la norma, parece que el legislador está prohibiendo con carácter general cualquier conducta que se realice y que tenga como consecuencia la falta de competencia aunque lo que realmente sanciona es cualquier conducta que pueda limitar el comportamiento de los agentes económicos según las reglas de competencia, no la falta de competencia en sí misma.

Al hilo del asunto que estamos abordando, el del sector del aceite de oliva y su problemática, conviene detenernos y analizar la posibilidad de que se establezcan y/o autoricen prácticas colusorias en el mercado. Esto es, acuerdos del sector del aceite que vayan encaminados a favorecer una estabilidad en los precios. Pues bien, es preciso que analicemos el apartado a y b del artículo 1.1 de la LDC que describe con precisión aquellas prácticas prohibidas que tienen más posibilidad de ponerse en práctica en el mercado, especialmente en el de productos agroalimentarios como el aceite de oliva y que dispone que están prohibidas "la fijación, de forma directa o indirecta, de precios o de otras condiciones comerciales o de servicio"13,

\footnotetext{
${ }^{8}$ Art. 169 del Reglamento (UE) $n^{\circ}$ 1308/2013, del Parlamento Europeo.

${ }^{9}$ Art. 169.2 c) del Reglamento (UE) no 1308/2013, del Parlamento Europeo.

${ }_{10}$ Art. 1 de la Ley 15/2007 de Defensa de la Competencia (en adelante LDC).

${ }^{11}$ Art. 2 de la LDC.

${ }_{12}$ Art. 3 de la LDC.

${ }^{13}$ Art. 1.1 a) de la LDC.
} 
y "la limitación o el control de la producción, la distribución, el desarrollo técnico o las inversiones" 14 .

Por tanto, ante un simple acuerdo del sector cooperativo de retirar parte de la producción de aceite de oliva del mercado o la simple recomendación de la no venta por parte de un grupo de comercializadores supondría según esta norma no sólo la nulidad del acuerdo adoptado tal cuál establece el artículo 1.2 de la LDC al disponer que: "son nulos de pleno derecho los acuerdos, decisiones y recomendaciones que, estando prohibidos en virtud de lo dispuesto en el apartado 1, no estén amparados por las exenciones previstas en la presente Ley" sino una infracción de la normativa de defensa de la competencia que llevaría aparejada una sanción tras la instrucción del oportuno expediente por parte de la Comisión Nacional de los Mercados y la Competencia.

Ahora bien, la prohibición que establece la norma para el veto de las prácticas colusorias no significa que sea determinante o excluyente, ya que el legislador ha previsto de forma taxativa una serie de medidas que permiten la práctica de determinados acciones y/o acuerdos colusorios siempre y cuando cumplan una serie de requisitos. A saber, la norma establece tres formas en las que se podría declarar la exención. Por un lado, especifica que es determinante que el acuerdo que se realice mejore la producción o la comercialización y que, además, esta decisión genere un efecto positivo en el mercado de manera que los consumidores puedan notar sus ventajas ${ }^{15}$. Por otro, establece la exención para las prácticas que sean aprobadas mediante instrumentos normativos tales como Reglamentos Comunitarios ${ }^{16}$ y por último, remite al caso en el que se apruebe un Real Decreto por parte del Gobierno en el que se determinen una serie de conductas que podrían estar permitidas ${ }^{17}$.

En particular y para el caso objeto de estudio, el gobierno español ha aprobado un Real Decreto que regula con detalle las normas sobre comercialización y producción de aceite de oliva.

\section{La limitación de la producción de los aceites de oliva a través del Real Decreto 84/2021, de 9 de febrero}

La entrada en vigor, el uno de julio, del Real Decreto 84/2021, de 9 de febrero, por el que se establecen las normas básicas para la aplicación del artículo 167 bis del Reglamento (UE) n. ${ }^{\circ}$ 1308/2013 del Parlamento Europeo

\footnotetext{
${ }^{14}$ Art. 1.1 b) de la LDC.

${ }^{15}$ Art. 1.3 de la LDC.

${ }^{16}$ Art. 1.4 de la LDC.

${ }^{17}$ Art. 1.5 de la LDC.
} 
y del Consejo, de 17 de diciembre de 2013, ha supuesto la regulación de las normas de comercialización del aceite de oliva ${ }^{18}$.

Este Real Decreto regula de forma breve pero precisa, cuáles son las condiciones y el contenido para activar esta norma de comercialización del aceite de oliva a nivel nacional en el momento en que la situación de mercado aconseje proceder a una estabilización. Siempre y cuando se cumpla con las condiciones establecidas en la norma y que son ajustadas a derecho y proporcionadas. Esta regulación no existe sólo en productos agroalimentarios como el aceite de oliva sino que sectores como el del vino ya viene aplicándola lo que permite anticiparse con determinadas decisiones a momentos de claro desequilibrio y perjuicio en el mercado para el sector.

Concretamente, esta norma permite que el sector oleícola fije determinadas medidas para impedir que haya un claro desequilibrio en el mercado. Las disposiciones son dos y desde el punto de vista de la eficacia y contundencia normativa se refieren a la retirada del aceite de oliva del mercado de forma temporal y/o ordenar destinarlo a uso no alimentario.

Pues bien, analizando el contenido de este Real Decreto, nos centraremos en primer lugar en el ámbito de aplicación recogido en el artículo 1 y que con carácter general hace referencia a todos los olivicultores de aceituna destinada a aceite de oliva, y a los operadores que elaboren, almacenen o comercialicen aceites de oliva en España ${ }^{19}$.

Una vez establecido los destinatarios que pueden ser objeto de dicha medida, la norma especifíca los requisitos para activar esta medida de comercialización en su artículo 3. En primer término y mediante una orden ministerial, previa audiencia a las organizaciones representativas del sector y de los responsables de las comunidades autónomas, se pueden establecer normas que regulen la oferta de aceite de oliva en el mercado con el objetivo de mejorar la estabilidad en los precios ${ }^{20}$. Esta orden ministerial se realizará cuando las condiciones del mercado lo justifiquen mediante criterios técnicos objetivos que no supongan un impacto negativo en la competencia, tal y como se aclara en la exposición de motivos. Resulta llamativo que esta última alusión no se realice en la parte dispositiva de la norma. En relación a esta cuestión, debemos traer a colación, que el Consejo de Estado, en su dictamen previo, determina "vagos e imprecisas" algunas cuestiones referentes a los criterios en virtud de los cuales el ministerio elegirá las regiones

18 BOE núm. 49 de 26 de febrero de 2021.

19 Art. 1 del Real Decreto 84/2021, de 9 de febrero, regulador de las normas de comercialización del aceite de oliva (en adelante, RD).

20 Art. 3.1 del RD. 
que se verán afectados por esta norma lo que podría provocar un conflicto entre territorios a la hora de su aprobación.

En segundo término la norma establece que dicha orden ministerial deberá optar por disponer la retirada del producto y/o el destino a uso no alimentario. Ahora bien, todo ello, correctamente justificado desde el punto de vista técnico, proporcional, analizando las condiciones del mercado y los efectos de su aplicación. Sorprende que el RD fije como plazo para aprobar la norma de comercialización con anterioridad al 31 de octubre, previa análisis de datos y audiencia a las regiones ${ }^{21}$. Aunque a priori pudiera parecer positivo enfrentarse a una campaña con una norma de comercialización ya aprobada por el Ministerio, desde nuestro punto de vista se debería tener en cuenta el hecho de que existen previsiones de producción que difieren de la realidad y que pudieran afectar a dicha norma. Esto es, si las previsiones de campaña se cifran en torno a 1,7 millones de toneladas y al término de la misma la cifra se reduce a 1,5, el hecho de que previamente se apruebe una norma que ante el aumento de la producción disponga la retirada del producto del mercado puede con posterioridad suponer la distorsión del mismo y el perjuicio en los precios.

Quizás resulte más adecuado, en este sentido, que la norma de comercialización se acomode al devenir real de una campaña determinada. Algo así parece que aclara el artículo 5 del RD cuando se refiere a la obligatoriedad de comunicar al Ministerio de Agricultura por parte de las Comunidades Autónomas, las estimaciones de existencias iniciales y las previsiones de producción para la campaña en cuestión antes del 30 de septiembre e, incluso, la comunicación durante la campaña de una variación al respecto ${ }^{22}$. Circunstancia que parece razonable y adecuado al objeto de que la norma de comercialización responda a la realidad. No obstante, queremos poner de manifiesto e incidir en lo que parece es un tema no resuelto ya que si el 31 de octubre, como plazo máximo para aprobar la norma de comercialización, se establece la cantidad de producto afectado para su retirada y/o destino a uso no alimentario y, posteriormente, las regiones comunican una variación en las cifras de producción la norma ya estará aprobada y no responderá al objetivo de estabilización del mercado del aceite de oliva.

Asimismo, el RD obliga a hacer constar en ella todos los datos que sirvan para justificar dicha medida y que están relacionados con la región o regiones afectadas por la norma ${ }^{23}$, la cantidad de producto afectado, la campaña a la que se aplicará, el objetivo que se persigue con la aprobación de la misma, los operadores económicos que se verán afectados así como

\footnotetext{
${ }^{21}$ Art. 3.3 del RD.

${ }^{22}$ Art. 5 del RD.

${ }^{23}$ Art. 4 del RD.
} 
los controles específicos y las normas para evaluar las medidas. Todo ello en aras a la transparencia y seguridad jurídica del sector. Sin lugar a dudas, ello supone acotar de forma taxativa, todas aquellas cuestiones que, de no ser determinadas, podrían dar lugar a equívocos en su aplicación. Véase como ejemplo el hecho de que el objetivo sea retirar producción en origen sólo en las cooperativas oleícolas de Andalucía y que la norma no determine tras su publicación el territorio o el tipo de empresa que se vería afectado por la misma. Esto supondría un claro perjuicio para el sector y la falta de un requisito fundamental para su correcta aplicación. Por ello, la falta de alguno de estos elementos desequilibraría la balanza en perjuicio de los operadores económicos en su conjunto.

En cuanto a los controles sobre lo dispuesto, el legislador lo deja en mano de las Comunidades Autónomas en coordinación con el Ministerio ${ }^{24}$. Aquí radica uno de los elementos más controvertidos en tanto la norma no prevé un sistema claro de supervisión y control. Por una parte, no se establece cuál sería el órgano que se encargue de coordinar entre las regiones y el estado la puesta en marcha de la norma y por otro, deja a los territorios que aplique conforme a sus normas como sería la comprobación de su cumplimiento por parte de los operadores económicos. Desde nuestro punto de vista, el establecimiento de un mecanismo de control claro y con un órgano competente a nivel nacional que coordine su puesta en marcha y su desarrollo sería mucho más efectivo al objeto de su cumplimiento.

El RD establece un régimen de infracciones y sanciones que se remite a las normas propias de cada comunidad autónoma y, en su caso, al Real Decreto por el que se regulan las infracciones y sanciones en materia de defensa del consumidor y de la producción agroalimentaria ${ }^{25}$. Esta norma ${ }^{26}$ establece como infracción en su artículo 3.3 apartado 3, el incumplimiento de las disposiciones administrativas sobre prohibición de comercializar determinados productos sin autorización administrativa. Quizás, en aras a una mayor simplificación normativa, hubiese sido conveniente determinar un régimen de infracciones y sanciones concreto para el caso que nos ocupa.

Por último, en cuánto al deber de información a las autoridades europeas el RD establece la obligatoriedad de comunicar a la Comisión el contenido de la norma de comercialización ${ }^{27}$. Esta exigencia deriva de un reglamento

${ }^{24}$ Art. 6 del RD.

${ }^{25}$ Art. 7 del RD.

${ }_{26}$ Real Decreto 1945/1983, de 22 de junio, por el que se regulan las infracciones y sanciones en materia de defensa del consumidor y de la producción agro-alimentaria, BOE núm. 168, de 15/07/1983.

${ }^{27}$ Art. 8 del RD. 
europe ${ }^{28}$ que establece en su articulado el deber de informar a la Comisión de todas las decisiones que adopten los estados miembros para mejorar el funcionamiento del mercado con el objetivo de regular la oferta ${ }^{29}$. Ahora bien, en ningún caso este reglamento hace alusión al sector del aceite de oliva sino al del vino. Por tanto, el estado español, ha dado un paso más allá de lo previsto en las disposiciones europeas aprobando una norma que en parte recoge lo que ya viene aplicándose en otros productos agroalimentarios como el vino.

\section{Conclusiones}

La inestabilidad en el sector del aceite de oliva, sobre todo en los precios en origen, requiere de una norma que tenga el objetivo de permitir una estabilización de precios del producto en origen. La Ley de Defensa de la Competencia permite con carácter excepcional establecer mediante Reales Decretos la posibilidad de adoptar la retirada o el destino del aceite de oliva a otro uso no alimentario con un doble objetivo, el de asegurar el valor añadido del aceite de oliva, y el mejor reparto de la renta social y territorial y la sostenibilidad de las explotaciones. La aprobación de esta norma supone un paso importante en la mejora de la comercialización del aceite de oliva al poner de manifiesto una nueva disposición perfectamente identificable no sólo para las administraciones, sino para el sector del olivar en general.

A la vista de lo anterior, creemos que una vía de solución a los problemas congénitos presentados por el mercado de los aceites de oliva pasa por la aplicación de este Real Decreto aunque, a priori, cabría pensar que permitir a los productores de aceite de oliva retirar de forma temporal el producto del mercado con el objetivo de subir los precios en origen cuando las circunstancias extraordinarias así lo justifiquen y aconsejen aprobar dicha medida podría suponer consagrar un práctica restrictiva de la competencia. Igualmente es posible cuestionarse si la norma protege o no a los consumidores finales que pueden verse perjudicados por la retirada de producto del mercado y el consiguiente aumento de los precios.

Desde nuestro punto de vista, este Real Decreto es bastante restrictivo $\mathrm{y}$ tiene en cuenta una serie de consideraciones tasadas que son aptas para

${ }^{28}$ Reglamento (UE) No 1308/2013 del Parlamento Europeo y del Consejo de 17 de diciembre de 2013 por el que se crea la organización común de mercados de los productos agrarios y por el que se derogan los Reglamentos (CEE) no 922/72, (CEE) no 234/79, (CE) no 1037/2001 y (CE) no $1234 / 2007$.

${ }^{29}$ Art. 167 del Reglamento (UE) No 1308/2013. 
legitimar la exención, tal como las normas comunitarias admiten. Desde la óptica de los consumidores entendemos que pueden beneficiarse de la estabilidad de precios tanto en los periodos de máxima oferta (al permitir su almacenamiento) como en los períodos de escasez cuando el aceite de oliva almacenado sea puesto en el mercado. Por lo que, sin ser una norma perfecta en su conjunto en cuanto al objetivo que persigue, representa una esperanza para el sector del olivar.

\section{BIBLIOGRAFÍA}

Girgado Perandones P. (2007), Grupo de Empresas y Derecho de Antitrust: la aplicación de las normas anticolusorias en las relaciones intragrupo, Madrid.

Gómez-Limón J.A., Parras Rosa M. (2017), Economía y comercialización de los aceites de oliva. Factores y perspectivas para el liderazgo español del mercado global, Cajamar. Jiménez Sánchez G. (2019), Lecciones de Derecho Mercantil, Madrid.

Miranda Serrano L. (2000), Restricciones accesorias y prácticas colusorias, "Derecho de los Negocios" nº 122.

Peinado Gracia J.I., Vázquez Ruano T. (2019), Tratado de Derecho de Sociedades Cooperativas, Valencia.

Sala Arquer J.M. (dir.) (2020), Comentario a la ley de defensa de la competencia, Madrid.

Sánchez Calero F. (2015), Instituciones de Derecho Mercantil, Navarra.

\section{THE LIMITATION OF THE PRODUCTION OF OLIVE OILS BY THE SPANISH STATE}

\section{Sum mary}

The instability of prices of produce at the source of its production in the agri-food sector presents a rather complicated situation. However, regarding the last link in the chain, namely marketing, the situation is quite different and this sector usually makes a profit on the sale of agricultural products. In Spain, the olive oil sector repeatedly suffers from this type of imbalance. Given these circumstances, the legislator has chosen to adopt, by means of a Royal Decree, some basic regulations that allow withdrawal of a certain production from the market in an orderly manner to alleviate the precarious situation, especially in terms of prices. This was done based on the European Union Regulation. In this article, the question that is asked is whether the operation of this regulation might lead to the distortion of the free market competition, or whether, perhaps, it may help to stabilise the prices at the source (original) production in the olive sector and thus protect the consumers.

Keywords: marketing, olive oil, collusive practices, antitrust 


\title{
LIMITI IMPOSTI ALLA PRODUZIONE DI OLI D'OLIVA DA PARTE DELLO STATO SPAGNOLO
}

\begin{abstract}
Riassunto
Il settore agroalimentare presenta, in generale, una situazione piuttosto complicata per quanto riguarda l'instabilità dei prezzi alla fonte. Tuttavia, con l'ultimo anello della catena, ovvero il marketing, la situazione è completamente diversa: questo settore, di solito, trae profitto dalla vendita dei prodotti agricoli. In Spagna, il settore dell'olio d'oliva soffre ripetutamente di questo tipo di squilibrio. Per cui, viste le circostanze, il legislatore ha scelto di approvare, con Regio Decreto, delle regole di base che consentano il ritiro ordinato della produzione dal mercato, al fine di alleviare la situazione precaria, soprattutto in termini di prezzi, e lo ha fatto sulla base di un regolamento dell'UE. Nell'articolo ci poniamo la domanda se il regolamento in questione possa portare a creare una instabilità concorrenziale sul mercato oppure se possa, forse, aiutare a stabilizzare i prezzi "alla fonte" del settore olivicolo, e quindi di tutelare i consumatori.
\end{abstract}

Parole chiave: marketing, olio d'oliva, pratiche collusive, antitrust 